\title{
THE EULER EQUATION FOR FUNCTIONALS WITH LINEAR GROWTH
}

BY

\author{
GABRIELE ANZELLOTTI
}

\begin{abstract}
We give a first variation formula for functionals of the type $\int_{\Omega} f(x, \mu)$, where $f(x, p): \Omega \times \mathbf{R}^{k} \rightarrow \mathbf{R}$ is of linear growth in $p$ for large $|p|$ and $\mu$ is a $\mathbf{R}^{k}$-valued measure in $\Omega$. The Euler equation for the minima of various functionals defined on spaces of BV. functions is then studied.
\end{abstract}

Introduction. Let us consider a functional of the type

$$
F(u)=\int_{\Omega} f(x, D u)+\int_{\Omega} H(x) u(x) d x,
$$

where $\Omega$ is an open bounded set in $\mathbf{R}^{n}, u: \Omega \rightarrow \mathbf{R}$ and $f(x, p): \Omega \times \mathbf{R}^{n} \rightarrow \mathbf{R}, H(x)$ : $\Omega \rightarrow \mathbf{R}$ are sufficiently nice. If a given function $u \in C^{2}(\Omega)$ is a local minimum for $F(u)$, then $u$ verifies the Euler equation

$$
D_{i} f_{p_{i}}(x, D u(x))=H(x) \text { in } \Omega \text {. }
$$

On the other hand, in the case that $f, H$ satisfy the assumptions

$$
|p| \leqslant f(x, p) \leqslant M(1+|p|) \text { for all } x, p,
$$

$f$ is continuous on $\Omega \times \mathbf{R}^{n}$,

$f$ is convex in $p$ for each fixed $x \in \Omega$,

$H \in L^{n}(\Omega)$ and it is sufficiently small,

the existence of minimum points with prescribed boundary conditions for suitable extensions [20, 15, 12, 13, 9] of functionals of the type considered is known [17, 15, $12,1]$ in the space $\mathrm{BV}(\Omega)=\left\{u \in L^{1}(\Omega) \mid D u\right.$ is a measure of bounded total variation\}. For the properties of BV functions we refer to $[19,23,11]$.

A typical example of a function $f(x, p)$ satisfying conditions (1.3) is the nonparametric area integrand $\sqrt{1+|p|^{2}}$, to which corresponds the functional

$$
F(u)=\int_{\Omega} \sqrt{1+|D u|^{2}}+\int_{\Omega} H(x) u(x) d x .
$$

Received by the editors January 5, 1984.

1980 Mathematics Subject Classification. Primary 49F10; Secondary 35D99, 26B30. 
Other examples, where we take $H=0$, are the functionals

$$
\int_{\Omega} \sqrt{1+a_{i j}(x) D_{i} u D_{j} u},
$$

where the functions $a_{i j}(x)$ are continuous and satisfy $a_{i j}(x)=a_{j i}(x),|\xi|^{2} \leqslant$ $a_{i j}(x) \xi_{i} \xi_{j} \leqslant M|\xi|^{2}$ for all $\xi \in \mathbf{R}^{n}$,

$$
\begin{gathered}
\int_{\Omega} \sqrt[k]{1+|D u|^{k}}, \quad k>1, \\
\int_{\Omega} \beta(|D u|), \text { where } \beta(t)= \begin{cases}t^{2} & \text { for } t \leqslant 1, \\
2 t-1 & \text { for } t \geqslant 1,\end{cases} \\
\int_{\Omega}|D u| .
\end{gathered}
$$

For the area functional $A(u)=\int_{\Omega} \sqrt{1+|D u|^{2}}$, it is well known $[17,24,14]$ that the local minima in $\mathrm{BV}(\Omega)$ are actually real analytic. The regularity $C^{2, \alpha}$ of the $\mathrm{BV}$ minima is known also for the functionals of the type (1.4) if the curvature $H$ is Lipschitz continuous $[18,8,29]$, and for the functional (1.5) if the coefficients $a_{i j}(x)$ are of class $C^{2, \alpha}$ [15]. Actually, the regularity $C^{2, \alpha}$ of the BV minima is proved in [15] for a whole class of functionals satisfying suitable structural conditions (essentially the conditions under which one is able to prove interior a priori bounds of the gradient for the smooth solutions of the corresponding Euler equation [22, 15, 26, 16]). In most cases, however (for instance for the functional (1.6)), the regularity of the local minima in BV is not known and, in many cases, one can easily construct examples of local minima which are discontinuous along an $(n-1)$-dimensional surface. This happens for instance for functionals (1.7) and (1.8), for (1.5) if the coefficients are not sufficiently smooth and for (1.4) if the curvature $H$ is for instance only $L^{\infty}$. Then it is natural to consider the problem of writing the Euler equation (1.2) in a suitable weak formulation that still holds for minima $u \in \mathrm{BV}(\Omega)$.

We get the following result: if the function $f(x, p)$ satisfies a few natural hypotheses of differentiability in $p$ (as in the assumptions of Theorem 3.6) and if $u \in \mathrm{BV}(\Omega)$ is a local minimum for $F(u)\left(\int_{\Omega} f(x, D u)\right.$ is defined for $\mathrm{BV}$ functions as in (3.1)), then one has

$$
\begin{aligned}
\left.\frac{d}{d t} F(u+t \varphi)\right|_{t=0}= & \int_{\Omega} H(x) \varphi(x) d x+\int_{\Omega} f_{p}\left(x,(D u)^{a}(x)\right) \cdot(D \varphi)^{a}(x) d x \\
& +\int_{\Omega} f_{p}^{0}\left(x, \frac{D u}{|D u|}(x)\right) \cdot \frac{D \varphi}{|D \varphi|}(x)|D \varphi|^{s} \\
= & 0
\end{aligned}
$$

for all admissible test functions $\varphi$, i.e. for all $\varphi \in \operatorname{BV}(\Omega)$ such that

$$
\begin{aligned}
& \operatorname{spt} \varphi \Subset \Omega, \\
& |D \varphi|^{s} \ll|D u|^{s}
\end{aligned}
$$

(i.e. $|D \varphi|^{s}$ is absolutely continuous with respect to $|D u|^{s}$ ), where $D u=(D u)^{a}+(D u)^{s}$ 
is the Lebesgue decomposition of the measure $D u$ in an absolutely continuous and a singular part with respect to the $n$-dimensional Lebesgue measure $\mathscr{L}^{n}$; we denote by $(D u)^{a}(x)$ the density of the measure $(D u)^{a}$ with respect to $\mathscr{L}^{n}$ and by $D u /|D u|$ the density of $D u$ with respect to its absolute variation $D u$. Finally, $f^{0}(x, p)$ is a positively homogeneous function in $p$, defined in (2.3), whose existence has to be assumed and that coincides with the recession function of $f(x, \cdot)$ in case $f$ is convex in $p$. For the notions of absolute continuity, Lebesgue decomposition and density (or derivative) of a measure $\lambda$ with respect to a positive measure $\mu$, we refer to [11, §2.9].

We recall that an equation of the type (1.9) has been introduced in [6] for the energy functional in Hencky plasticity.

The key point in (1.9) is that the test functions $\varphi$ are allowed to have "jumps", but only where the minimum $u$ itself has a "jump". The fact of considering variations $\varphi \in \mathrm{BV}(\Omega)$ is important, because equation (1.9), if it is considered only for all functions $\varphi \in C_{0}^{\infty}(\Omega)$ (that is for $\varphi \in H_{0}^{1,1}(\Omega)$ ), does not carry all the information related to the fact that $u$ is a local minimum (Remark 3.12). On the other hand, the restriction $(1.10, \mathrm{ii})$ is natural in two ways: first, the derivative $\left.(d / d t) F(u+t \varphi)\right|_{t=0}$ exists if and only if $(1.10$,ii) holds; second, under natural assumptions on $f$, if a function $u \in \mathrm{BV}(\Omega)$ verifies equation (1.9) for all the admissible test functions, then $u$ is a local minimum for $F(u)$ (Theorem 3.10).

The validity of equation (1.9) is also proved, for a restricted class of admissible variations, if $f$ is not differentiable in $p$ at the points $p$ where $f$ takes the value zero (Theorem 3.9). This result applies in particular to the case where $f(x, p)$ is positively homogeneous in $p$, as for the functional (1.8).

The results on the differentiability of the functional $F(u)$ are obtained from corresponding results, given in $\S 2$, for the functional $I(\mu)=\int_{\Omega} f(x, \mu)$, where $\mu$ is a general $\mathbf{R}^{k}$-valued Borel measure in $\Omega$. One could use the general results of $\S 2$ also to get an Euler equation for functionals of the type

$$
\int_{\Omega} f(x, \varepsilon(u))
$$

where $u \in L^{1}\left(\Omega, \mathbf{R}^{n}\right)$ is a vector field of bounded deformation, i.e. the distributions $\varepsilon_{i j}(u)=\frac{1}{2}\left[D_{i} u^{j}+D_{j} u^{i}\right]$ are measures of bounded total variation in $\Omega$ [27]. However, we shall not develop such a theory, as it is totally similar to the one given in $\$ 3$ for $F(u)$.

In $\$ 2$ we collect also a few simple facts about the extension to the space of measures of the functional $I(\eta)=\int f(x, \eta(x)) d x$. We shall adopt the approach of $[25,15,13,9]$. Recent papers [28, 10] give an extension by making use of convex analysis and duality theory. In fact, most examples of integrands $f(x, p)$ in the calculus of variations are convex in $p$, on the other hand the convexity is not needed for many of our purposes and we shall assume it only when it is necessary.

We conclude with a few remarks.

First: if $u \in \operatorname{BV}(\Omega)$ is a local minimum for the functional (1.1) and we write equation (1.9) for all $\varphi \in C_{0}^{\infty}(\Omega)$, then we get that the vector field $\varphi(x)=$ $f_{p}\left(x,(D u)^{a}(x)\right)$ satisfies $\operatorname{div} \psi=H$ and $\psi$ belongs to the class $X(\Omega)_{n}=\{\psi \in$ $\left.L^{\infty}(\Omega) \mid \operatorname{div} \psi \in L^{n}(\Omega)\right\}$ considered in [2]. This fact has been one of the motivations for the work done in [2]. 
Second: Once we have the Euler equation for the functional $F(u)$, it will be natural to try a standard technique to get information on the regularity of a local minimum $u$ of $F$, i.e. to write equation (1.9) for the test functions of the type $\varphi=\eta u$, where $\eta \in C_{0}^{\infty}(\Omega)$ (that certainly satisfy condition (1.10)). This has been done already for the energy functional in Hencky plasticity [3], compare [21], and it will be done in a forthcoming paper for general functionals of the type (1.1).

Third: For functions in $\mathrm{BV}(\Omega)$ the Euler equation (1.9) suggests the possibility of a new weak formulation for the quasilinear equations of the type $\operatorname{div} A(x, D u)=$ $B(x)$, where $A(x, p): \Omega \times \mathbf{R}^{n} \rightarrow \mathbf{R}^{n}$ and $B(x): \Omega \rightarrow \mathbf{R}$, which are not necessarily the Euler equation of any functional.

I would like to thank G. Dal Maso for some useful conversations about the semicontinuity results in [9].

2. Directional derivatives of the functional $I(\mu)=\int_{\Omega} f(x, \mu)$. First, we are going to define a real valued Borel measure $f(x, \mu)$ on $\Omega$ for all functions $f(x, p): \Omega \times \mathbf{R}^{k} \rightarrow \mathbf{R}$ that satisfy a few suitable conditions and for all $\mu \in M\left(\Omega, \mathbf{R}^{k}\right)$, where $M\left(\Omega, \mathbf{R}^{k}\right)$ denotes the space of the $\mathbf{R}^{k}$-valued Borel measures in $\Omega$.

Then we shall consider the functional $I(\mu)=\int_{\Omega} f(x, \mu)$ and we shall obtain a formula for the derivative $\left.(d / d t) I(\mu+t \beta)\right|_{t=0}$ under suitable assumptions on $f$ and $\beta$.

Let us consider the following conditions:

$$
f(x, p) \geqslant 0,
$$

$f$ is such that for all Borel measurable functions $\eta: \Omega \rightarrow \mathbf{R}^{k}$, the function $x \rightarrow f(x, \eta(x))$ is Borel measurable,

for all $x \in \Omega$ there exists the finite limit

$$
\lim _{t \rightarrow 0^{+}} f\left(x, \frac{p}{t}\right) t=f^{0}(x, p) \text {. }
$$

We remark that condition (2.2) is satisfied for instance when $f$ is a Borel function in $(x, p)$. Condition (2.3) says that the function $f$ is asymptotically linear for large $p$.

It is immediately evident that the function $f^{0}(x, p)$ is positively homogeneous in $p$, i.e.

$$
f^{0}(x, s p)=s f^{0}(x, p) \text { for all } x, p \text { and } s>0,
$$

and that $f^{0}(x, p)$ satisfies condition (2.2) if $f$ does.

Now we can give the

DEFINITION 2.1. Let $f$ satisfy conditions (2.1), (2.2) and (2.3). Then for any measure $\mu \in M\left(\Omega, \mathbf{R}^{k}\right)$ we define a positive measure $f(x, \mu)$ on $\Omega$ as

$$
\int_{B} f(x, \mu)=\int_{B} f\left(x, \mu^{a}(x)\right) d x+\int_{B} f^{0}\left(x, \frac{d \mu^{s}}{d|\mu|^{s}}(x)\right) d|\mu|^{s}
$$

for all Borel sets $B \subset \Omega$, where $\mu=\mu^{a}+\mu^{s}$ is the Lebesgue decomposition of the measure $\mu$ in an absolutely continuous and a singular part with respect to the Lebesgue measure $\mathscr{L}^{n}$. We denote by $\mu^{a}(x)$ the density of the measure $\mu^{a}$ with respect to $\mathscr{L}^{n}$ and by $\left(d \mu^{s} / d|\mu|^{s}\right)(x)$ the density of $\mu^{s}$ with respect to $|\mu|^{s}$. 
In formula (2.4) we could write $(d \mu / d|\mu|)(x)$ instead of $\left(d \mu^{s} / d|\mu|^{s}\right)(x)$, because the two functions are equal $|\mu|^{s}$-a.e.

If the function $f$ is such that

$$
|f(x, p)| \leqslant c(1+|p|)
$$

then one also has $\left|f^{0}(x, p)\right| \leqslant c|p|$ and

$$
\int_{\Omega} f(x, \mu) \leqslant c\left(\mathscr{L}^{n}(\Omega)+|\mu|(\Omega)\right)
$$

hence, if (2.2), (2.3), (2.5) hold and $|\mu|(\Omega), \mathscr{L}^{n}(\Omega)$ are finite, then formula (2.4) defines a measure $f(x, \mu)$ also without imposing condition (2.1), and the measure $f(x, \mu)$ has in this case a finite total variation.

We should like to remark that one could easily extend the preceding definitions to more general measures $\mu$ and to other positive measures than $\mathscr{L}^{n}$. We also remark that for a measure $\mu=\eta(x) \mathscr{L}^{n}$, which is absolutely continuous with respect to $\mathscr{L}^{n}$, one has $\int_{B} f(x, \mu)=\int_{B} f(x, \eta(x)) d x$, while for a singular measure $\mu=\mu^{s}$ we have

$$
\int_{B} f(x, \mu)=\int_{B} f^{0}\left(x, \frac{d u}{d|\mu|}(x)\right) d|\mu| .
$$

We shall use the following simple fact.

LeMMA 2.2. If $f(x, p)$ is positively homogeneous in $p$, then for all Borel sets $B \subset \Omega$, one has

(i) $\int_{B} f\left(x, \frac{d \mu}{d|\mu|}(x)\right) d|\mu|=\int_{B} f\left(x, \frac{d \mu_{1}}{d\left|\mu_{1}\right|}(x)\right) d\left|\mu_{1}\right|+\int_{B} f\left(x, \frac{d \mu_{2}}{d\left|\mu_{2}\right|}(x)\right) d\left|\mu_{2}\right|$,

$$
\text { if } \mu_{1}+\mu_{2}=\mu \text { and } \mu_{1}, \mu_{2} \text { are mutually singular, and }
$$

$$
\int_{B} f(x, \mu)=\int_{B} f\left(x, \frac{d \mu}{d \alpha}(x)\right) d \alpha
$$

if $\alpha$ is a positive measure such that $\mu \ll \alpha$.

Proof. As $\mu_{1}$ and $\mu_{2}$ are mutually singular, we have $|\mu|=\left|\mu_{1}\right|+\left|\mu_{2}\right|$; hence

$$
\int_{B} f\left(x, \frac{d \mu}{d|\mu|}(x)\right) d|\mu|=\int_{B} f\left(x, \frac{d \mu}{d|\mu|}(x)\right) d\left|\mu_{1}\right|+\int_{B} f\left(x, \frac{d \mu}{d|\mu|}(x)\right) d\left|\mu_{2}\right|
$$

and (i) follows because one also has that

$$
\begin{aligned}
& \frac{d \mu}{d|\mu|}(x)=\frac{d \mu_{1}}{d|\mu|}(x)=\frac{d \mu_{1}}{d\left|\mu_{1}\right|}(x) \quad\left|\mu_{1}\right| \text {-a.e. in } \Omega, \\
& \frac{d \mu}{d|\mu|}(x)=\frac{d \mu_{2}}{d|\mu|}(x)=\frac{d \mu_{2}}{d\left|\mu_{2}\right|}(x) \quad\left|\mu_{2}\right| \text {-a.e. in } \Omega .
\end{aligned}
$$

Now, writing $\mu=\mu^{a}+\mu^{s}$, by (2.4) and statement (i) we get immediately that

$$
\begin{aligned}
\int_{B} f(x, \mu) & =\int_{B} f\left(x, \frac{d \mu}{d|\mu|}(x)\right) d|\mu| \\
& =\int_{B} f\left(x, \frac{(d \mu / d \alpha)(x)}{(d|\mu| / d \alpha)(x)}\right) \frac{d|\mu|}{d \alpha} d \alpha
\end{aligned}
$$

and (ii) follows because of the homogeneity of $f$ in $p$. Q.E.D. 
Now we shall see another way of writing the measure $f(x, \mu)$ which has a useful geometrical meaning when $\mu=D u$ and $u \in \operatorname{BV}(\Omega)$. Let us consider the function $\tilde{f}(x, p, t): \Omega \times \mathbf{R}^{k} \times \mathbf{R}^{k} \times[0,+\infty) \rightarrow \mathbf{R}$ defined as

$$
\bar{f}(x, p, t)= \begin{cases}f(x, p / t) t & \text { if } t>0 \\ f^{0}(x, p) & \text { if } t=0\end{cases}
$$

One then has

THEOREM 2.3. Assume that $f$ satisfies conditions (2.2), (2.3), (2.5) and that $|\mu|(\Omega), \mathscr{L}^{n}(\Omega)$ are finite. Then one has

$$
\int_{B} f(x, \mu)=\int_{B} \bar{f}\left(x, \frac{d \mu}{d \alpha}(x), \frac{d \mathscr{L}^{c}}{d \alpha}(x)\right) d \alpha,
$$

where $\alpha$ is any positive Borel measure such that $|\mu|+\mathscr{L}^{n} \ll \alpha$.

Proof. First, it is easy to see that for any pair of Borel functions $\eta: \Omega \rightarrow \mathbf{R}^{k}$ and $\xi$ : $\Omega \rightarrow[0,+\infty)$, the function $x \rightarrow \bar{f}(x, \eta(x), \xi(x))$ is also Borel measurable; hence the integral to the right in (2.7) is well defined. Consider then the $\mathbf{R}^{k} \times \mathbf{R}$ valued measures $\lambda=\left(\mu, \mathscr{L}^{n}\right), \lambda_{1}=\left(\mu^{a}, \mathscr{L}^{n}\right)$ and $\lambda_{2}=\left(\mu^{s}, 0\right)$. We have that $\lambda_{1}+\lambda_{2}=\lambda$ and that $\lambda_{1}, \lambda_{2}$ are mutually singular; hence, by Lemma 2.2 we get

$$
\begin{aligned}
\int_{B} \bar{f}\left(x, \frac{d \mu}{d|\lambda|}(x), \frac{d \mathscr{L}^{n}}{d|\lambda|}(x)\right) d|\lambda| \\
\quad=\int_{B} \bar{f}\left(x, \frac{d \mu^{a}}{d|\lambda|}(x), \frac{d \mathscr{L}^{n}}{d|\lambda|}(x)\right) d\left|\lambda_{1}\right|+\bar{f}\left(x, \frac{d \mu^{s}}{d|\lambda|}(x), 0\right) d\left|\lambda_{2}\right| \\
\quad=\int_{B} \bar{f}\left(x, \frac{d \mu^{a}}{d \mathscr{L}^{n}}(x), 1\right) d \mathscr{L}^{n}+\int_{B} f^{0}\left(x, \frac{d \mu^{s}}{d\left|\mu^{s}\right|}(x)\right) d\left|\mu^{s}\right|
\end{aligned}
$$

which proves (2.7) for the particular choice $\alpha=|\lambda|$. The proof for general $\alpha$ follows by statement (ii) of Lemma 2.2. Q.E.D.

Now we shall study the differentiability of $I(\mu)$. From now on, we shall assume that $f$ satisfies (2.2), (2.3), (2.5), that $\mathscr{L}^{n}(\Omega)<+\infty$ and we shall only consider measures $\mu, \beta \in M\left(\Omega, \mathbf{R}^{k}\right)$ of finite total variation.

THEOREM 2.4. Assume that $f(x, p)$ is differentiable in $p$ for all $(x, p) \in \Omega \times \mathbf{R}^{k}$ and that $f^{0}(x, p)$ is differentiable in $p$ for all $(x, p) \in \Omega \times\left(\mathbf{R}^{k}-\{0\}\right)$. Assume also that

$$
\left|f_{p}(x, p)\right| \leqslant M, \quad\left|f_{p}^{0}(x, p)\right| \leqslant M .
$$

Then the functional $I(\mu)$ is differentiable at the point $\mu$ in the direction $\beta$ if and only if $|\beta|^{s} \ll|\mu|^{s}$ and in such a case one has

$$
\begin{aligned}
\left.\frac{d}{d t} I(\mu+t \beta)\right|_{t=0}= & \int_{\Omega} f_{p}\left(x, \mu^{a}(x)\right) \cdot \beta^{a}(x) d x \\
& +\int_{\Omega} f_{p}^{0}\left(x, \frac{d \mu^{s}}{d|\mu|^{s}}(x)\right) \cdot \frac{d \beta^{s}}{d|\beta|^{s}}(x) d \beta^{s} .
\end{aligned}
$$


Proof. Let $\mu$ be fixed. For each $\beta$ we consider the Lebesgue decomposition $\beta^{s}=\beta^{s a}+\beta^{s s}$ of the measure $\beta^{s}$ in an absolutely continuous and a singular part with respect to $|\mu|^{s}$. Then we have

$$
\mu+t \beta=\mu^{a}+\mu^{s}+t \beta^{a}+t \beta^{s a}+t \beta^{s s}
$$

and, using Lemma 2.2(i) and (ii), we get

$$
\begin{aligned}
I(\mu+t \beta)= & \int_{\Omega} f\left(x, \mu^{a}(x)+t \beta^{a}(x)\right) d x \\
& +\int_{\Omega} f^{0}\left(x, \frac{d \mu^{s}}{d|\mu|^{s}}(x)+t \frac{d \beta^{s a}}{d|\mu|^{s}}(x)\right) d|\mu|^{s}+|t| \int_{\Omega} f^{0}\left(x, \beta^{s s}\right) .
\end{aligned}
$$

Now we obviously have that

$$
\left.\frac{d}{d t} \int_{\Omega} f\left(x, \mu^{a}(x)+t \beta^{a}(x)\right) d x\right|_{t=0}=\int_{\Omega} f_{p}\left(x, \mu^{a}(x)\right) \cdot \beta^{a}(x) d x .
$$

Moreover, as $f^{0}(x, p)$ is differentiable in $p$ for $p \neq 0$ and $\left|\left(d \mu^{s} / d|\mu|^{s}\right)(x)\right|=1$ $|\mu|^{s}$-a.e., we have

$$
\begin{gathered}
\left.\frac{d}{d t} \int_{\Omega} f^{0}\left(x, \frac{d \mu^{s}}{d|\mu|^{s}}(x)+t \frac{d \beta^{s a}}{d|\mu|^{s}}(x)\right) d|\mu|^{s}\right|_{t=0} \\
=\int_{\Omega} f_{p}^{0}\left(x, \frac{d \mu^{s}}{d|\mu|^{s}}(x)\right) \cdot \frac{d \beta^{s a}}{d|\mu|^{s}}(x) d|\mu|^{s} \\
=\int_{\Omega} f_{p}^{0}\left(x, \frac{d \mu^{s}}{d|\mu|^{s}}(x)\right) \cdot \frac{d \beta^{s a}}{d\left|\beta^{s a}\right|} d\left|\beta^{s a}\right|
\end{gathered}
$$

and we also have

$$
\left.\frac{d^{+}}{d t}\left(|t| \int_{\Omega} f^{0}\left(x, \beta^{s s}\right)\right)\right|_{t=0}=\int_{\Omega} f^{0}\left(x, \beta^{s s}\right)=-\left.\frac{d^{-}}{d t}\left(|t| \int_{\Omega} f^{0}\left(x, \beta^{s s}\right)\right)\right|_{t=0} .
$$

It is evident that the right and left derivatives in the last formula coincide and are then zero if and only if $\int_{\Omega} f^{0}\left(x, \beta^{s s}\right)=0$, i.e. if and only if $\beta^{s s}=0$, that is if and only if $\beta^{s} \ll|\mu|^{s}$. Q.E.D.

The preceding theorem does not apply when the integrand $f(x, p)$ is positive and positively homogeneous in $p$, because in this case $f(x, p)$ cannot be differentiable in $p$ at $p=0$. Also in this case, however, we can differentiate the functional $I(\mu)$ in a suitably restricted set of directions. More generally, we have the following result.

THEOREM 2.5. Assume that for every fixed $x \in \Omega$ the function $f(x, p)$ is differentiable in $p$ for all $p \neq 0$ and assume that $f(x, 0)=0$ for all $x$ such that $f(x, \cdot)$ is not differentiable at $p=0$. Assume also that $f^{0}(x, p)$ is differentiable in $p$ for all $(x, p) \in \Omega \times\left(\mathbf{R}^{k}-\{0\}\right)$. Finally assume that

$$
\left|f_{p}(x, p)\right| \leqslant M, \quad\left|f_{p}^{0}(x, p)\right| \leqslant M
$$


when the derivatives exist. Then, if $\mu, \beta$ are such that

$$
\beta^{s} \ll|\mu|^{s},
$$

$$
\beta^{a}(x)=0 \quad \mathscr{L}^{n} \text {-a.e. in the set } T=\left\{x \in \Omega \mid \mu^{a}(x)=0\right\},
$$

the functional $I(\mu)$ is differentiable at the point $\mu$ in the direction $\beta$ and formula (2.9) still holds.

Proof. We start again from (2.10), where $\beta^{s s}=0$ by $(2.12$, i). For all $x \in \Omega \backslash T$ we have

$$
\left.\frac{d}{d t} f\left(x, \mu^{a}(x)+t \beta^{a}(x)\right)\right|_{t=0}=f_{p}\left(x, \mu^{a}(x)\right) \cdot \beta^{a}(x) .
$$

On the other hand, because of $\left(2.12\right.$, ii), for $\mathscr{L}^{n}$-almost all $x \in T$ we have $\beta^{a}(x)=0$ and

$$
\frac{d}{d t} f\left(x, \mu^{a}(x)+t \beta^{a}(x)\right) \equiv 0 .
$$

Formula (2.9) follows then as in the preceding theorem. Q.E.D.

We remark that conditions (2.12) are equivalent to saying that $\beta \ll|\mu|$.

3. The Euler equation in $\mathrm{BV}(\Omega)$. In this section we shall consider integrands $f(x, p): \Omega \times \mathbf{R}^{n} \rightarrow \mathbf{R}$ that, unless it is otherwise specified, satisfy conditions (2.2), (2.3), (2.5), and we shall apply the results of the preceding section to the functional

$$
F(u)=\int_{\Omega} f(x, D u)=\int_{\Omega} f\left(x,(D u)^{a}(x)\right) d x+\int_{\Omega} f^{0}\left(x, \frac{D u}{|D u|}(x)\right)|D u|^{s}
$$

which is defined for all functions $u \in \operatorname{BV}(\Omega)$; we assume that $\Omega \subset \mathbf{R}^{n}$ is bounded.

The functional $F(u)$ is an extension to the space $\operatorname{BV}(\Omega)$ of the functional $\int_{\Omega} f(x, D u(x)) d x$, which is defined elementarily for $u \in C^{1}(\Omega) \cap H^{1,1}(\Omega)$. The interest for considering this extension stems mainly from a few known facts that we would like to recall briefly here for both completeness and later reference.

FACT 3.1. Assume that the function $\bar{f}(x, p, t)$ defined in (2.6) is continuous. Then, if $u_{j} \in \mathrm{BV}(\Omega)$ and

$$
\begin{aligned}
u_{j} & \rightarrow u \text { in } L^{1}(\Omega), \\
\int_{\Omega} \sqrt{1+\left|D u_{j}\right|^{2}} & \rightarrow \int_{\Omega} \sqrt{1+|D u|^{2}} \quad \text { for } j \rightarrow \infty,
\end{aligned}
$$

one also has $\int_{\Omega} f\left(x, D u_{j}\right) \rightarrow \int_{\Omega} f(x, D u)$.

FACT 3.2. Assume that $\bar{f}(x, p, t)$ is continuous, and that it is convex in $(p, t)$ for all fixed $x \in \Omega$. Then, if $u, u_{j} \in \mathrm{BV}(\Omega)$ are such that

$$
\begin{gathered}
u_{j} \rightarrow u \text { in } L^{1}(\Omega), \\
\int_{\Omega}\left|D u_{j}\right| \leqslant C \quad \text { for all } j
\end{gathered}
$$


one also has

$$
\liminf _{j \rightarrow+\infty} \int_{\Omega} f\left(x, D u_{j}\right) \geqslant \int_{\Omega} f(x, D u) .
$$

The proof of Facts 3.1, 3.2 is an immediate consequence of Theorem 2.3 and of Theorems 3 and 2 of [25], which are given for general functionals of a measure (and would apply also to the functional (1.11) [5]).

It is worth recalling that under the assumption of convexity in $p$ and (2.5), a necessary and sufficient condition on $f(x, p)$ in order for $\bar{f}(x, p, t)$ to be continuous is that [7] for all $x \in \Omega$ and $\varepsilon>0$ there exists a number $\delta>0$ such that

$$
\left|f\left(x_{0}, p\right)-f(x, p)\right|<\varepsilon(1+|p|)
$$

for all $p$ and all $x$ with $\left|x-x_{0}\right|<\delta$. We recall also (same proof as in [4, Theorem 1]) that for all the functions $u \in \operatorname{BV}(\Omega)$ there exists a sequence $u_{j} \in C^{\infty}(\Omega) \cap \operatorname{BV}(\Omega)$ such that (3.2) holds.

It is not difficult to see that Fact 3.2 holds under the weaker assumption that $\bar{f}$ is lower-semicontinuous; and we recall that if $f(x, p)$ is lower-semicontinuous, convex in $p$ and satisfies (2.5), then $\bar{f}(x, p, t)$ is also lower-semicontinuous. Condition (3.3, ii) can be dropped if one assumes also that $f(x, p) \geqslant a|p|-b$ for all $x \in \Omega$, $p \in \mathbf{R}^{k}$, where $a>0, b \geqslant 0$. As a general reference for these and other more general semicontinuity results, see [9]. See also the recent paper [30].

In conclusion, if $\bar{f}(x, p, t)$ is continuous and convex in $(p, t)$ and $f(x, p) \geqslant a|p|$ $-b$ for all $x, p$, then one has

$$
\int_{\Omega} f(x, D u)=\min \left\{\liminf _{j \rightarrow \infty} f\left(x, D u_{j}(x)\right) d x \mid \begin{array}{l}
u_{j} \in C^{1}(\Omega) \cap \mathrm{BV}(\Omega), \\
u_{j} \rightarrow u \quad \text { in } L^{1}(\Omega)
\end{array}\right\},
$$

compare $[13,9]$.

If we assume that $\Omega$ has a Lipschitz boundary and that $f(x, p)$ is defined also for $x \in \partial \Omega$, we can consider the functional $[15,13,1]$

$$
G(u)=\int_{\Omega} f(x, D u)+\int_{\partial \Omega} f^{0}\left(x, \nu_{\Omega}(x)[g(x)-u(x)]\right) d H^{n-1},
$$

where $g \in L^{1}(\partial \Omega)$ is a given function and $\nu_{\Omega}(x)$ is the outward unit normal to $\partial \Omega$ at $x$. Again one easily has (appendix):

FACT 3.3. Assume that the function $\bar{f}(x, p, t)$ is continuous in $\bar{\Omega} \times \mathbf{R}^{n} \times[0,+\infty)$. Then, for all $u \in \mathrm{BV}(\Omega)$ and $g \in L^{1}(\partial \Omega)$ there exists a sequence of functions $u_{j} \in$ $C^{1}(\Omega) \cap \mathrm{BV}(\Omega)$ such that $\left.u_{j}\right|_{\partial \Omega}=g$ and that

$$
u_{j} \rightarrow u \text { in } L^{1}(\Omega), \quad G\left(u_{j}\right) \rightarrow G(u) .
$$

FACT 3.4. Assume that $\bar{f}(x, p, t)$ is lower-semicontinuous on $\bar{\Omega} \times \mathbf{R}^{n} \times[0,+\infty)$ and is convex in $(p, t)$ for each fixed $x \in \bar{\Omega}$, and that $f(x, p) \geqslant a|p|-b$ for all $x, p$. Then, for any fixed $g \in L^{1}(\partial \Omega)$ and for any sequence $u_{j} \in \mathrm{BV}(\Omega)$ such that $u_{j} \rightarrow u$ in $L^{1}(\Omega)$ one has

$$
\liminf _{j \rightarrow \infty} G\left(u_{j}\right) \geqslant G(u)
$$


By Facts 3.3 and 3.4 immediately follows

FACT 3.5. If $\bar{f}(x, p, t)$ satisfies the assumptions of Facts 3.3 and 3.4, then $G(u)$ is the greatest functional on $\mathrm{BV}(\Omega)$ which is lower-semicontinuous with respect to the $L^{1}(\Omega)$-convergence and satisfies $G(u) \leqslant \int_{\Omega} f(x, D u(x)) d x$ for all functions $u \in C^{1}(\Omega)$ $\cap H^{1,1}(\Omega)$ with $u=g$ on $\partial \Omega$. Moreover one has

$$
\inf _{u \in \operatorname{BV}(\Omega)} G(u)=\inf _{\substack{u \in \operatorname{BV}(\Omega) \cap C^{1}(\Omega) \\ u=g \text { on } \partial \Omega}} \int_{\Omega} f(x, D u(x)) d x .
$$

The preceding considerations motivate the study of the problem

$$
G(u) \rightarrow \min , \quad u \in \operatorname{BV}(\Omega) .
$$

Now we shall study the differentiability and the Euler equation of the functionals $F(u)$ and $G(u)$. For these purposes we shall not need any convexity and global continuity assumptions on the integrand $f(x, p)$. Of course, convexity and continuity will be needed in Theorem 3.10 .

THEOREM 3.6. Assume that $f(x, p)$ is differentiable in $p$ for all $x \in \Omega, p \in \mathbf{R}^{n}$ and assume that $f^{0}(x, p)$ is differentiable for all $x \in \Omega, p \in \mathbf{R}^{n}-\{0\}$. Assume moreover that

$$
\left|f_{p}(x, p)\right| \leqslant M, \quad\left|f_{p}^{0}(x, p)\right| \leqslant M .
$$

Then the functional $F(u)$ is differentiable at the point $u \in \operatorname{BV}(\Omega)$ in the direction $\varphi \in \operatorname{BV}(\Omega)$ if and only if $|D \varphi|^{s} \ll|D u|^{s}$, and in such a case one has

$$
\begin{aligned}
\left.\frac{d}{d t} F(u+t \varphi)\right|_{t=0}= & \int_{\Omega} f_{p}\left(x,(D u)^{a}(x)\right) \cdot(D \varphi)^{a}(x) d x \\
& +\int_{\Omega} f_{p}^{0}\left(x, \frac{D u}{|D u|}(x)\right) \cdot \frac{D \varphi}{|D \varphi|}(x)|D \varphi|^{s} .
\end{aligned}
$$

Theorem 3.6 follows immediately from Theorem 2.4. By Theorem 3.6 we get

THEOREM 3.7. Let $\Omega$ be a bounded open set in $\mathbf{R}^{n}$ with Lipschitz boundary. Assume that $f(x, p): \bar{\Omega} \times \mathbf{R}^{n} \rightarrow \mathbf{R}$ is differentiable in $p$ for all $(x, p)$, that $f^{0}(x, p)$ is differentiable in $p$ for all $(x, p)$ with $p \neq 0$ and that

$$
\left|f_{p}(x, p)\right| \leqslant M, \quad\left|f_{p}^{0}(x, p)\right| \leqslant M
$$

(recall also that we are always assuming (2.2), (2.3), (2.5)). If $u \in \mathrm{BV}(\Omega)$ is a minimum point for the functional $G(v)$ defined in (3.3), then one has

$$
\begin{aligned}
\left.\frac{d}{d t} G(u+t \varphi)\right|_{t=0}= & \int_{\Omega} f_{p}\left(x,(D u)^{a}(x)\right) \cdot(D \varphi)^{a}(x) d x \\
& +\int_{\Omega} f_{p}^{0}\left(x, \frac{D u}{|D u|}(x)\right) \cdot \frac{D \varphi}{|D \varphi|}(x)|D \varphi|^{s} \\
& -\int_{\partial \Omega} f_{p}^{0}\left(x, \frac{g(x)-u(x)}{|g(x)-u(x)|} \nu_{\Omega}(x)\right) \cdot \nu_{\Omega}(x) \varphi(x) d H^{n-1} \\
= & 0
\end{aligned}
$$


for all the functions $\varphi \in \mathrm{BV}(\Omega)$ such that

$$
\begin{gathered}
|D \varphi|^{s} \ll|D u|^{s}, \\
\varphi(x)=0 \quad H^{n-1} \text {-a.e. in the set } T_{1}=\{x \in \partial \Omega \mid u(x)=g(x)\} .
\end{gathered}
$$

Proof. One can use Theorem 3.6 to differentiate $\int_{\Omega} f(x, D u)$. Because of the homogeneity of $f^{0}$, for the boundary term one has

$$
\begin{aligned}
f^{0}\left(x, \nu_{\Omega}(x)[(g(x)-\right. & u(x))-t \varphi(x)]) \\
& =f^{0}\left(x, \frac{g(x)-u(x)}{|g(x)-u(x)|} \nu_{\Omega}(x)\right)|(g(x)-u(x))-t \varphi(x)|
\end{aligned}
$$

for all $x \in \partial \Omega$ where $u(x) \neq g(x)$, and for sufficiently small $t$, it follows that

$$
\begin{aligned}
& \left.\frac{d}{d t} \int_{\partial \Omega} f^{0}\left(x, \nu_{\Omega}(x)[g(x)-(u(x)+t \varphi(x))]\right) d H^{n-1}\right|_{t=0} \\
& \quad=-\int_{\partial \Omega} f^{0}\left(x, \frac{g(x)-u(x)}{|g(x)-u(x)|} \nu_{\Omega}(x)\right) \frac{g(x)-u(x)}{|g(x)-u(x)|} \varphi(x) d H^{n-1} \\
& =-\int_{\partial \Omega} f_{p}^{0}\left(x, \frac{g(x)-u(x)}{|g(x)-u(x)|} \nu_{\Omega}(x)\right) \cdot \nu_{\Omega}(x) \varphi(x) d H^{n-1} \text {. Q.E.D. }
\end{aligned}
$$

REMARK 3.8. It is clear that for the differentiability of the boundary term one needs only the directional derivative of $f^{0}(x, p)$ at each point $p \neq 0$ in the radial direction $p$, and this derivative always exists because of the homogeneity of $f^{0}$. A similar fact happens in the interior of $\Omega$ in the set $N(u)$ defined in (4.4).

Clearly one can use Theorem 2.5 to get the Euler equation for the case of positive homogeneous integrands. We have

THEOREM 3.9. Assume that $f$ satisfies the assumptions of Theorem 2.5 and let $u$ be a minium point for the functional $G$. Then, for all the functions $\varphi=\mathrm{BV}(\Omega)$ such that

$$
|D \varphi|^{s} \ll|D u|^{s}
$$

$$
\begin{gathered}
(D \varphi)^{a}(x)=0 \quad \mathscr{L}^{n} \text {-a.e. in the set } T=\left\{x \in \Omega \mid(D u)^{a}(x)=0\right\}, \\
\varphi(x)=0 \quad H^{n-1} \text {-a.e. in the set } T_{1}=\{x \in \partial \Omega \mid u(x)=g(x)\},
\end{gathered}
$$

the equation (3.6) holds.

We shall say that the set of equations (3.6) for all the functions that satisfy (3.7) is the Euler equation in $\operatorname{BV}(\Omega)$ for the functional $G(u)$. This Euler equation does not state the vanishing of the first variation of $G$ in all directions $\varphi$, nevertheless, we still have the following result.

THEOREM 3.10. Let $f(x, p)$ be as in Theorem 3.7 and assume moreover that

$$
\begin{gathered}
f(x, p) \text { is convex in } p \text { for all fixed } x \in \Omega, \\
\bar{f}(x, p, t) \text { is continuous on } \bar{\Omega} \times \mathbf{R}^{n} \times[0,+\infty) .
\end{gathered}
$$

If a function $v \in \mathrm{BV}(\Omega)$ verifies equation (3.6) for all the functions $\varphi \in \mathrm{BV}(\Omega)$ that satisfy (3.7), then $v$ is a minimum point for the functional $G(u)$ in $\mathrm{BV}(\Omega)$. 
Proof. Take a sequence of functions $v_{j} \in C^{1}(\Omega) \cap H^{1,1}(\Omega)$ such that $\left.v_{j}\right|_{\partial \Omega}=g$ for all $j$, and that converge to $v$ as in (3.4). For any fixed $j$ set $\varphi_{j}=v_{j}-u$ and consider the function $H(s):[0,1] \rightarrow \mathbf{R}$ defined by $H(s)=G\left(u+t \varphi_{j}\right)$. By (3.9) one has that $\bar{f}(x, p, t)$ is convex in $(p, t)$ and it follows that $H(s)$ is a convex function; hence one also has

$$
G\left(v_{j}\right)=H(1) \geqslant H(0)+H(0)=G(u)+\left.\frac{d}{d t} G\left(u+t \varphi_{j}\right)\right|_{t=0}=G(u),
$$

where we have used (3.6), as $\varphi_{j}$ satisfies (3.7). By (3.11) and (3.4) the theorem is proved. Q.E.D.

A condition like (3.10) is needed in the hypotheses of Theorem 3.10, as the following example shows.

EXAmple 3.11. Consider the open set $\Omega=\{x \in \mathbf{R} \mid 0<x<3\}$ and define a function $f(x, p): \Omega \times \mathbf{R} \rightarrow \mathbf{R}$ as

$$
f(x, p)= \begin{cases}\sqrt{1+p^{2}} & \text { if } x \neq 1, x \neq 2, \\ c^{-1} \sqrt{1+p^{2}} & \text { if } x=1, \\ \alpha \sqrt{1+p^{2}} & \text { if } x=2,\end{cases}
$$

where $c$ is any number such that $3<c<\sqrt{10}$ and $0<\alpha<c-3$. Take also $g(0)=0$ and $g(s)=1$. Then the function $v(x)$ defined as

$$
v(x)= \begin{cases}\frac{1}{3} \sqrt{c^{2}-9} x & \text { if } 0<x<1 \\ \frac{1}{3} \sqrt{c^{2}-9} x+\left(1-\sqrt{c^{2}-9}\right) & \text { if } 1<x<3\end{cases}
$$

satisfies equation (3.6) for all the functions $\varphi \in \mathrm{BV}(\Omega)$ such that (3.7) holds: in fact, for all these functions one has $G(v) \leqslant G(v+\varphi)$. On the other hand, $v$ is not a minimum for $G(u)$, because, if we set for instance

$$
w(x)= \begin{cases}0 & \text { if } 0<x<2 \\ 1 & \text { if } 2<x<3\end{cases}
$$

we have

$$
G(w)=3+\alpha<c<c+\frac{1}{c}\left(1-\sqrt{c^{2}}-9\right)=G(v)
$$

REMARK 3.12. In Theorem 3.10 we have assumed that $v$ verifies equation (3.6) for all the variations that satisfy (3.7). To assume that (3.6) is satisfied for all $\varphi \in H^{1,1}(\Omega)$ would not have been sufficient, in fact, consider the following one-dimensional example:

$$
\begin{gathered}
\Omega=\{x \in \mathbf{R} \mid 0<x<2\} ; f(x, p)=\sqrt{1+p^{2}} ; g(0)=0, \quad g(1)=1, \\
v(x)= \begin{cases}0 & \text { if } 0<x<1, \\
1 & \text { if } 1<x<2 .\end{cases}
\end{gathered}
$$

It is clear that $(D v)^{a}=0$ and $f_{p}\left(x,(D u)^{a}(x)\right)=0$ for all $x$; on the other hand, for all $\varphi \in H_{0}^{1,1}(\Omega)$ one has $(D \varphi)^{s}=0$ and equation (3.6) is satisfied, while $v$ certainly does not minimize length. 
Clearly, similar results to Theorem 3.10 hold for functionals of type (1.11) and for local minima.

As a final general remark for this section, we notice that one could study by similar methods also the subdifferentials of the functionals $F(u)$ and $G(u)$.

4. The functionals $f(x, u, D u)$. Let $\Omega$ be an open bounded set in $\mathbf{R}^{n}$ and consider a function $f(x, s, p): \Omega \times \mathbf{R} \times \mathbf{R}^{n} \rightarrow \mathbf{R}$ such that

$$
|f(x, s, p)| \leqslant c(1+|p|)
$$

for all $(x, s) \in \Omega \times \mathbf{R}$ there exists the limit

$$
\lim _{t \downarrow 0^{+}} f\left(x, s, \frac{p}{t}\right) t=f^{0}(x, s, p),
$$

for all Borel measurable functions $\eta: \Omega \rightarrow \mathbf{R}^{n}$ and $\xi: \Omega \rightarrow \mathbf{R}$, the function $x \rightarrow f(x, \xi(x), \eta(x))$ is also Borel measurable.

For each function $u \in \operatorname{BV}(\Omega)$ we consider a Borel set $M(u)$ such that $\mathscr{L}^{n}(M(u))$ $=|D u|^{s}(\Omega \backslash M(u))=0$. Following $[11,9]$ we set

$$
\begin{aligned}
& u_{-}(x)=\sup \left\{t \mid\{y \mid u(y)<t\} \text { has } \mathscr{L}^{n} \text {-density zero at } x\right\}, \\
& u_{+}(x)=\inf \left\{t \mid\{y \mid u(y)>t\} \text { has } \mathscr{L}^{n} \text {-density zero at } x\right\}, \\
& N(u)=\left\{x \in \Omega \mid u_{+}(x)>u_{-}(x)\right\}
\end{aligned}
$$

and we remark that $u_{-}(x), u_{+}(x)$ are Borel measurable functions on $\Omega$. Moreover we shall consider the measures

$$
\begin{aligned}
& D u^{c}=\left.D u\right|_{N(u)}=\left.\left(u_{+}(x)-u_{-}(x)\right) \frac{D u}{|D u|}(x) H^{n-1}\right|_{N(u)}, \\
& D u^{d}=(D u)^{s}-D u^{c}=\left.D u\right|_{M(u) \backslash N(u)}
\end{aligned}
$$

and we shall need the following simple fact (appendix):

FACT 4.1. If $\varphi, u \in \mathrm{BV}(\Omega)$ are such that $|D \varphi|^{s} \ll|D u|^{s}$, then one has

$$
\begin{gathered}
\left|D \varphi^{c}\right| \ll\left|D u^{c}\right|, \\
\left|D \varphi^{d}\right| \ll\left|D u^{d}\right|, \\
H^{n-1}(N(\varphi) \backslash N(u))=0, \\
\frac{D \varphi}{|D \varphi|}(x)=\sigma(x) \frac{D u}{|D u|}(x) \quad H^{n-1} \text {-a.e. in } N(\varphi),
\end{gathered}
$$

where $\sigma(x)= \pm 1$.

Finally, we consider the functional [9]

$$
\begin{aligned}
F(u)= & \int_{\Omega} f(x, u, D u) \\
= & \int_{\Omega} f\left(x, u(x),(D u)^{a}(x)\right) d x+\int_{\Omega} f^{0}\left(x, u(x), \frac{D u}{|D u|}(x)\right)|D u|^{d} \\
& +\int_{N(u)} \int_{u_{-}(x)}^{u_{+}(x)} f^{0}\left(x, s, \frac{D u}{|D u|}(x)\right) d s d H^{n-1}
\end{aligned}
$$


and the functional [1]

$$
G(u)=F(u)+\int_{\partial \Omega} \int_{u(x)}^{g(x)} f^{0}\left(x, s, \nu_{\Omega}(x)\right) d s d H^{n-1}
$$

where $g(x) \in L^{1}(\partial \Omega)$ is some fixed function and $\nu_{\Omega}$ is the outward normal to $\partial \Omega$.

For the functional $G(u)$ we have completely similar results to Facts 3.3, 3.4, 3.5; the reader himself can provide the statements and proofs. Now we give the Euler equation for $F(u)$, a similar result holds for $G(u)$.

THEOREM 4.2. Assume that:

(i) for all $(x, s, p)$ the function $f$ is differentiable in $s$ and in $p$, and the function $f^{0}$ is differentiable in $s$; moreover one has

$$
\left|f_{p}(x, s, p)\right|+\left|f_{s}(x, s, p)\right|+\left|f_{s}^{0}(x, s, p)\right| \leqslant M
$$

(ii) the function $f^{0}$ is differentiable in $p$ for all $(x, s, p)$ with $p \neq 0$, and one has $\left|f_{p}^{0}(x, s, p)\right| \leqslant M$,

(iii) $u \in \mathrm{BV}(\Omega)$ is a local minimum for $F(u)$.

Then, for all the functions $\varphi \in \operatorname{BV}(\Omega)$ with spt $\varphi \Subset \Omega$ such that $|D \varphi|^{s} \ll|D u|^{s}$, one has

$$
\begin{aligned}
& \left.\frac{d}{d t} F(u+t \varphi)\right|_{t=0} \\
& =\int_{\Omega}\left\{f_{s}\left(x, u(x),(D u)^{a}(x)\right) \varphi(x)+f_{p}\left(x, u(x),(D u)^{a}(x)\right)(D \varphi)^{a}(x)\right\} d x \\
& \quad+\int_{\Omega}\left\{f_{s}^{0}\left(x, u(x), \frac{D u}{|D u|}(x)\right) \varphi(x)+f_{p}^{0}\left(x, u(x), \frac{D u}{|D u|}(x)\right) \frac{D \varphi}{|D \varphi|}(x)\right\}|D u|^{d} \\
& +\int_{N^{+}(u)}\left\{f^{0}\left(x, u_{+}(x), \frac{D u}{|D u|}(x)\right) \varphi_{+}(x)\right. \\
& \quad+\int_{N^{-}(u)}\left\{f^{0}\left(x, u_{+}(x), \frac{D u}{|D u|}(x)\right) \varphi_{-}(x)\right. \\
&
\end{aligned}
$$

$=0$, 
where

$$
\begin{aligned}
& N^{+}(u)=\left\{x \in N(u) \cap N(\varphi) \mid \frac{D u}{|D u|}(x)=\frac{D \varphi}{|D \varphi|}(x)\right\}, \\
& N^{-}(u)=\left\{x \in N(u) \cap N(\varphi) \mid \frac{D u}{|D u|}(x)=-\frac{D \varphi}{|D \varphi|}(x)\right\} .
\end{aligned}
$$

Proof. Write $F(u+t \varphi)$ using formula (4.6). The derivative of the term containing $(D u+t D \varphi)^{a}$ is easily computed. For the term containing $\left|D(u+t \varphi)^{d}\right|$, recalling that $\left|D(u+t \varphi)^{d}\right| \ll\left|D u^{d}\right|$, that one has $(D u /|D u|)(x)=\left(D u^{d} /\left|D u^{d}\right|\right)(x)\left|D u^{d}\right|$-a.e., and using the homogeneity of $f^{0}$ in $p$, we get, recalling Lemma 2.2(ii),

$$
\begin{aligned}
\int_{\Omega} f^{0} & \left(x, u(x)+t \varphi(x), \frac{D(u+t \varphi)}{|D(u+t \varphi)|}(x)\right)\left|D(u+t \varphi)^{d}\right| \\
& =\int_{\Omega} f^{0}\left(x, u(x)+t \varphi(x), \frac{D u}{|D u|}(x)+t \frac{D \varphi}{|D u|}(x)\right)\left|D u^{d}\right|
\end{aligned}
$$

and the derivative is again easily obtained as in (2.11).

Finally, recalling Fact 4.1, one has

$$
\begin{gathered}
\int_{N(u+t \varphi)} \int_{(u+t \varphi)_{-}}^{(u+t \varphi)_{+}} f^{0}\left(x, s, \frac{D(u+t \varphi)}{|D(u+t \varphi)|}(x)\right) d s d H^{n-1} \\
=\int_{N(u)} \int_{(u+t \varphi)_{-}}^{(u+t \varphi)_{+}} f^{0}\left(x, s, \sigma_{t}(x) \frac{D u}{|D u|}(x)\right) d s d H^{n-1}
\end{gathered}
$$

because $N(u+t \varphi) \subset N(u)$ and $(u+t \varphi)_{+}=(u+t \varphi)_{-}$exactly at those points of $N(u)$ that do not belong to $N(u+t \varphi)$.

Now it is easy to see that for a fixed $x \in N(u)$ one has $\sigma_{t}(x)=+1$ for all $t$ that belong to some neighborhood of zero. Moreover for all these $t$ one has

$$
\begin{aligned}
& \left.\begin{array}{l}
(u+t \varphi)_{+}=u_{+}+t \varphi_{+} \\
(u+t \varphi)_{-}=u_{-}+t \varphi_{-}
\end{array}\right\} \quad \text { if } \frac{D u}{|D u|}(x)=\frac{D \varphi}{|D \varphi|}(x), \\
& \left.\begin{array}{l}
(u+t \varphi)_{+}=u_{+}+t \varphi_{-} \\
(u+t \varphi)_{-}=u_{-}+t \varphi_{+}
\end{array}\right\} \quad \text { if } \frac{D u}{|D u|}(x)=-\frac{D \varphi}{|D \varphi|}(x)
\end{aligned}
$$

and it follows that

$$
\begin{aligned}
& \frac{d}{d t} \int_{(u+t \varphi)_{-}}^{(u+t \varphi)_{+}} f^{0}\left(x, s, \frac{D u}{|D u|}(x)\right) d s \\
& =\left\{\begin{array}{l}
f^{0}\left(x, u_{+}(x), \frac{D u}{|D u|}(x)\right) \varphi_{+}(x)-f^{0}\left(x, u_{-}(x), \frac{D u}{|D u|}(x)\right) \varphi_{-}(x) \text { in } N^{+}(u), \\
f^{0}\left(x, u_{+}(x), \frac{D u}{|D u|}(x)\right) \varphi_{-}(x)-f^{0}\left(x, u(x), \frac{D u}{|D u|}(x)\right) \varphi_{+}(x) \text { in } N^{-}(u) .
\end{array}\right.
\end{aligned}
$$

Q.E.D. 
The assumption in Theorem 4.2 that $f_{s}(x, s, p)$ is bounded is somewhat too restrictive, in fact it is not satisfied for example by the functionals of the type $\int a(u) \sqrt{1+|D u|^{2}}$ in which case one has $f_{s}(x, u, p)=a^{\prime}(u) \sqrt{1+|p|^{2}}$. A more natural assumption would be

$$
\left|f_{s}(x, s, p)\right| \leqslant M(1+|p|) .
$$

In fact, similarly to what happens for the functionals of growth $m>1$, one is still able to write the Euler equation under assumption (4.9) provided one takes bounded test functions. More precisely, one has

THEOREM 4.3. Assume that:

(i) for all $(x, s, p)$ the function $f$ is differentiable in $s$ and $p$ and the function $f^{0}$ is differentiable in $s$; moreover one has

$$
\left|f_{p}(x, s, p)\right| \leqslant M, \quad\left|f_{s}(x, s, p)\right| \leqslant M(1+|p|), \quad\left|f_{s}^{0}(x, s, p)\right| \leqslant M|p|,
$$

(ii) the function $f^{0}$ is differentiable in $p$ for all $(x, s, p)$ with $p \neq 0$, and one has $\left|f_{p}^{0}(x, s, p)\right| \leqslant M$,

(iii) $u \in \mathrm{BV}(\Omega)$ is a local minimum for $F(u)$.

Then, for all the functions $\varphi \in \mathrm{BV}(\Omega)$ such that

one has (4.8).

$$
\operatorname{spt} \varphi \Subset \Omega, \quad\|\varphi\|_{\infty}<+\infty, \quad|D \varphi|^{s} \ll|D u|^{s},
$$

The proof of Theorem 4.3 is totally similar to the proof of Theorem 4.2.

We notice that similar results to Theorem 3.9 also hold for the functionals considered in this section.

A general final remark about functionals of type $\int_{\Omega} f(x, u, D u)$ is that the integrand could be allowed not to be defined or not to satisfy the assumptions of our theorems in a set $E_{0} \subset \Omega_{x} \times \mathbf{R}_{s}$ of zero $H^{n}$-dimensional measure (see, for example, [9]). This is a consequence of the general fact that if $u \in \operatorname{BV}(\Omega)$ and $B \subset \Omega$ with $H^{n-1}(B)=0$, then one has $\int_{B}|D u|=0$.

\section{Appendix.}

Proof of FACT 3.3. Let $v_{j} \in C^{1}(\Omega) \cap \mathrm{BV}(\Omega)$ be a sequence of functions that converge to $u$ as in (3.2) and such that $v_{j}=u$ on $\partial \Omega$ for all $j$ [19]. Then let $w_{j} \in C^{1}(\Omega) \cap \operatorname{BV}(\Omega)$ be such that, for each $j$, one has

$$
\begin{aligned}
& \left.w_{j}\right|_{\partial \Omega}=g-\left.u\right|_{\partial \Omega}, \quad \dot{w}_{j}(x)=0 \quad \text { if } \operatorname{dist}(x, \partial \Omega)<\frac{1}{j}, \\
& \int_{\Omega}\left|D w_{j}\right| \leqslant \int_{\partial \Omega}|u-g| d H^{n-1}+\frac{1}{j}, \quad \int_{\Omega}\left|w_{j}\right| \leqslant \frac{1}{j} .
\end{aligned}
$$

Set $u_{j}=v_{j}+w_{j}$. Obviously, one has $\left.u_{j}\right|_{\partial \Omega}=g$ for all $j$ and

$$
\begin{gathered}
u_{j} \rightarrow u \text { in } L^{1}(\Omega), \\
\lim _{j \rightarrow \infty} \int_{\Omega} \sqrt{1+\left|D u_{j}\right|^{2}}=\int_{\Omega} \sqrt{1+|D u|^{2}}+\int_{\partial \Omega}|u-g| d H^{n-1} .
\end{gathered}
$$


If we consider the $\mathbf{R}^{n}$-valued measures $\mu_{j}, \mu$ on $\bar{\Omega}$ defined by

$$
\mu_{j}(B)=\int_{B \cap \Omega} D u_{j}, \quad \mu(B)=\int_{B \cap \Omega} D u+\int_{B \cap \partial \Omega}(g-u) \nu_{\Omega}(x) d H^{n-1}
$$

for all Borel sets $B \subset \bar{\Omega}$, and the $\mathbf{R}^{n+1}$-valued measures

$$
\alpha_{j}(B)=\left(\mu_{j}(B), \mathscr{L}^{n}(B)\right), \quad \alpha(B)=\left(\mu(B), \mathscr{L}^{n}(B)\right),
$$

then we have

$$
\alpha_{j} \rightarrow \alpha \quad \text { weakly as measures in } \bar{\Omega}, \quad \lim _{j \rightarrow \infty}\left|\alpha_{j}\right|(\bar{\Omega})=|\alpha|(\bar{\Omega}) .
$$

Now we notice that

$$
G(u)=\int_{\bar{\Omega}} \bar{f}(x, \alpha), \quad G\left(u_{j}\right)=\int_{\bar{\Omega}} \bar{f}\left(x, \alpha_{j}\right)
$$

and the proof is concluded by Theorem 3 of [25]. Q.E.D.

Proof of FACT 3.4. Let $\Omega_{1}$ be some open ball containing $\bar{\Omega}$ and consider the function $f^{*}(x, p): \Omega_{1} \times \mathbf{R}^{n} \rightarrow \mathbf{R}$ defined as

$$
f^{*}(x, p)= \begin{cases}f(x, p) & \text { if } x \in \bar{\Omega}, \\ M(1+|p|) & \text { if } x \in \Omega_{1} \backslash \bar{\Omega} .\end{cases}
$$

By (2.5) one also has that $f^{*}(x, p)$ is lower-semicontinuous. Let $h \in H^{1,1}\left(\Omega_{1}\right)$ be such that $\left.h\right|_{\partial \Omega}=g$ and for each function $u \in \operatorname{BV}(\Omega)$ consider the function $u^{*} \in$ $\operatorname{BV}\left(\Omega_{1}\right)$ defined as

$$
u^{*}(x)= \begin{cases}u(x) & \text { if } x \in \Omega, \\ h(x) & \text { if } x \in \Omega_{1} \backslash \Omega .\end{cases}
$$

For all $u \in \mathrm{BV}(\Omega)$ one has

$$
\int_{\Omega_{1}} f^{*}\left(x, D u^{*}\right)=G(u)+M \int_{\Omega_{1} \backslash \bar{\Omega}}(1+|D h|)=G(u)+\operatorname{const}(h)
$$

and the proof is concluded noting that if $u_{j} \rightarrow u$ in $L^{1}(\Omega)$ one also has $u_{j}^{*} \rightarrow u^{*}$ in $L^{1}\left(\Omega_{1}\right)$ and using Fact 3.2 for $f^{*}$ in $\Omega_{1}$. Q.E.D.

Proof OF FACT 4.1. If $|D \varphi|^{s} \ll|D u|^{s}$ one has $D \varphi=h|D u|^{s}$, where $h(x)=$ $\left((D \varphi)^{s} /|D u|^{s}\right)(x)$, and it follows that $D \varphi^{c}=\left.\left(h|D u|^{s}\right)\right|_{N(\varphi)}=h|(D u)|_{N(\varphi)}$ so that $(4.5, \mathrm{i})$ is proved.

Now, if we set $N_{k}(\varphi)=\left\{x \in N(\varphi) \mid \varphi_{+}(x)-\varphi_{-}(x)>1 / k\right\}$ for $k$ a positive integer, we have $N(\varphi) \backslash N(u)=\bigcup_{k} N_{k}(\varphi) \backslash N(u)$ and, if we assume by contradiction that $H^{n-1}(N(\varphi) \backslash N(u))>0$, we get

$$
|D \varphi|\left(N_{k}(\varphi) \backslash N(u)\right)>\frac{1}{k} H^{n-1}\left(N_{k}(\varphi) \backslash N(u)\right)>0
$$

for some $k$. This contradicts $(4.5, \mathrm{i})$ and proves $(4.5$, iii).

Then, if $\left|D u^{d}\right|(B)=0$ for some Borel set $B \subset \Omega$, one has $|D u|^{s}(B \backslash N(u))=0$ and, by the assumption that $|D \varphi|^{s} \ll|D u|^{s}$, it follows that $|D \varphi|^{s}(B \backslash N(u))=0$; hence we get $|D \varphi|^{d}(B \backslash N(u))=0$. On the other hand one has $\left|D \varphi^{d}\right|(N(u))=$ $|D \varphi|(N(u) \backslash N(\varphi))=0$ and $(4.5$, ii) also follows. 
Finally, we recall that $[11,4.5 .9], N(u)$ and $N(\varphi)$ are $\left(H^{n-1}, n-1\right)$-countably rectifiable sets, so that $[11,3.2 .19]$ there exists a Borel set $E_{0}$ such that $H^{n-1}\left(E_{0}\right)=0$ and such that for all $x \in N(\varphi) \backslash E_{0}$ the sets $\operatorname{Tan}\left(H^{n-1} \angle N(\varphi), x\right)$ and $\operatorname{Tan}\left(H^{n-1} \angle N(u), x\right)[11,3.2 .16]$, are $(n-1)$-dimensional subspaces of $\mathbf{R}^{n}$. On the other hand, from $\left(4.5\right.$, iii) it follows obviously that $\operatorname{Tan}\left(H^{n-1} \angle N(\varphi), x\right) \subset$ $\operatorname{Tan}\left(H^{n-1} \angle N(u), x\right)$ for $H^{n-1}$-almost all $x \in N(\varphi)$; hence the two tangent planes must coincide for $H^{n-1}$-almost all $x \in N(\varphi)$. As $(D \varphi /|D \varphi|)(x)$ and $(D u /|D u|)(x)$ are normal vectors to $\operatorname{Tan}\left(H^{n-1} \angle N(\varphi), x\right)$ and $\operatorname{Tan}\left(H^{n-1} \angle N(u), x\right)$ respectively, $(4.5, \mathrm{iv})$ is proved. Q.E.D.

\section{REFERENCES}

1. G. Anzellotti, Dirichlet problem and removable singularities for functionals with linear growth, Boll. Un. Mat. Ital. C (5) 28 (1981), 141-159.

2. __ Pairings between measures and bounded functions and compensated compactness, Ann. Mat. Pura Appl. (4) 135 (1983), 293-318.

3. __ On the extremal stress and displacement in Hencky plasticity, Duke Math. J. 51 (1984), $133-147$.

4. G. Anzellotti and M. Giaquinta, Funzioni BV e tracce, Rend. Sem. Mat. Padova 60 (1978), 1-21.

5. __ Existence of the displacement field for an elasto-plastic body subject to Hencky's law and Von Mises yield condition, Manuscripta Math. 32 (1980), 101-136.

6. . On the existence of the fields of stresses and displacements for an elasto-perfectly plastic body in static equilibrium, J. Math. Pures Appl. 61 (1982), 219-244.

7. M. Boni, Quasi additività e quasi subadditività nell'integrale ordinario del calcolo delle variazioni alla Weierstrass, Rend. Istit. Mat. Univ. Trieste 6 (1974), 1-20.

8. E. Bombieri and E. Giusti, Local estimates for the gradient of nonparametric surfaces of prescribed mean curvature, Comm. Pure Appl. Math. 26 (1973), 381-394.

9. G. Dal Maso, Integral representation on $\mathrm{BV}(\Omega)$ of $\Gamma$-limits of variational integrals, Manuscripta Math. 30 (1980), 387-416.

10. F. Demengel and R. Temam, Convex function of a measure and applications, Indiana J. Math. (to appear).

11. H. Federer, Geometric measure theory, Springer-Verlag, Berlin and New York, 1969.

12. R. Ferro, Variational functionals defined on spaces of BV functions and their dependence on boundary data, Ann. Mat. Pura Appl. 122 (1979), 269-287.

13. Integral characterization of functionals defined on spaces of BV functions, Rend. Sem. Mat. Univ. Padova 61 (1979), 177-201.

14. C. Gerhardt, On the regularity of solutions to variational problems in BV( $\Omega)$, Math. Z. 149 (1976), $281-286$.

15. M. Giaquinta, G. Modica and J. Soucek, Functionals with linear growth in the calculus of variations, Comm. Math. Univ. Carolinae 20 (1979), 143-171.

16. D. Gilbarg and N. S. Trudinger, Elliptic P.D.E. of second order, Springer-Verlag, Berlin and Heidelberg, 1977.

17. E. Giusti, Superfici cartesiane di area minima, Rend. Sem. Mat. Fis. Milano 40 (1970), 3-21.

18. Boundary value problems for non-parametric surfaces of prescribed mean curvature, Ann. Scuola Norm. Sup. (4) 3 (1976), 501-548.

19. Minimal surfaces and functions of bounded variation, Notes on Pure Math. 10, A.C.T., Canberra, 1977. (A revised edition is going to be published by Birkhäuser.)

20. C. Goffman and J. Serrin, Sublinear functions of measures and variational integrals, Duke Mat. J. 31 (1964), 159-178.

21. R. Hardt and D. Kinderlehrer, Elastic plastic deformation, Univ. of Minnesota Math. reports, 1983.

22. O. A. Ladizenskaia and N. N. Ural' tseva, Local estimates for gradients of solutions of non-uniformly elliptic and parabolic equations, Comm. Pure Appl. Math. 83 (1970), 677-703.

23. U. Massari and M. Miranda, Minimal surfaces of codimension one monograph, Notas de Matematica. North-Holland, Amsterdam, 1984.

24. M. Miranda, Un principio di massimo forte per le frortiere minimali, Rend. Sem. Mat. Padova 45 (1971), 355-356. 
25. Yu. G. Reschetniak, Weak convergence of completely additive vector functions on a set, Sibirsk. Mat. Ž. 9 (1968), 1386-1394. (Translated)

26. L. Simon, Interior gradient bounds for non-uniformly elliptic equations, Indiana Univ. Math. J. 25 (1976), 821-855.

27. G. Strang and R. Temam, Functions of bounded deformation, Arch. Rational Mech. Anal. 75 (1980), $7-21$.

28. R. Temam, Approximation de fonction convexes sur un espace de mesures et applications, Canad. Math. Bull. (4) 25 (1982), 392-413.

29. N. S. Trudinger, Gradient estimates and mean curvature, Math. Z. 131 (1973), 165-175.

30. E. De Giorgi, G. Buttazzo and G. Dal Maso, On the lower semicontinuity of certain integral functionals, Rend. Accad. Naz. Lincei (1984).

Dipartimento di Matematica, Universita di Trento, 38050 Povo (TN), Italia 